\title{
THREE DIMENSIONAL PLANNING OF ORTHOGNATHIC SURGERY: A NARRATIVE REVIEW OF THE LEUVEN PROTOCOL
}

\author{
Eman Shaheen ${ }^{1,2 a}$ iD , Constantinus Politis $^{1,2 b^{*}}$ iD \\ 1OMFS IMPATH research group, Department of Imaging \& Pathology, Faculty of Medicine, KU Leuven, Leuven, Belgium \\ ${ }^{2}$ Oral and Maxillofacial Surgery, University Hospitals Leuven, Leuven, Belgium \\ a BSc, MSc, PhD, Clinical Engineer, Responsible \\ ${ }^{\mathrm{b}}$ MD, DDS, MHA, MM, PhD, Professor and Head of Department of Oral and Maxillofacial Surgery
}

ABSTRACT DOI: https://doi.org/10.25241/stomaeduj.2019.6(3).art.4

Background \& Objective: The aim of this paper was to present the virtual surgical planning (VSP) protocol for orthognathic surgery used in daily practice in the department of Oral and Maxillofacial surgery, University Hospitals of Leuven, Leuven, Belgium.

Data Sources: The different steps are presented in details explaining the protocol and showing an example of a bimaxillary case.

Data Synthesis: A variety of aspects have been discussed including the different possibilities with respect to the software used and their limitations. The pros and cons of that software compared to other commercial software tools have been highlighted.

Keywords: 3D planning; Orthognathic surgery; Virtual planning; Surgical planning; Virtual surgical planning (VSP).

\section{Introduction}

Surgical virtual planning is currently possible due to the recent developments in maxillofacial radiology with the introduction of low dose cone beam computer tomography (CBCT) especially with $\mathrm{CBCT}$ systems scanning the full skull. The added value of virtual planning of orthognathic surgery has been proven which include a better and more accurate outcome as stated by Stokbro et al in their systematic review [1]. The virtual planning is significantly faster for single- and double-jaw surgery compared to the conventional method [2]. Moreover, Scheinder et al. recommended the use of virtual model surgery and the prefabricated three dimensionally (3D) printed splints to replace traditional orthognathic surgery as it becomes cost-effective [3]. Several virtual planning protocols have been published in the recent years [4-9]. One protocol was called the triple scan CBCT protocol [9] as it relied on 3 CBCT scans: first the patient is scanned with a thin wax bite in the mouth, which is followed by a limited dose scan with a tray of impression in the mouth. Then the last step is a high resolution scan of the patient's tray of impression. That protocol had the privilege of automating some preprocessing steps to allow for faster planning. However, this protocol has been replaced with the commonly used protocol with only one CBCT of the patient's full skull with a thin wax bite. A variety of software programs are available commercially implementing the one-scan protocol,

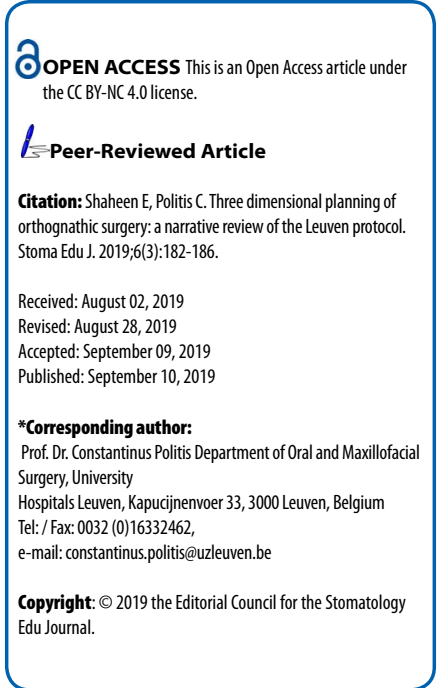

commonly known as Virtual Surgical Planning (VSP), but with different algorithms and tools whether semi or fully automated. In this paper, we present the VSP protocol used in University Hospitals of Leuven to virtually plan orthognathic surgery in daily practice after introducing improvements.

\section{Methodology}

The VSP protocol can be divided into 10 steps which will be explained in details in this section based on the Proplan software (Materialise, Leuven, Belgium) as used in the department of Oral and Maxillofacial surgery, University Hospitals of Leuven, Leuven, Belgium. There is a wizard specifically made for orthognathic VSP. An example is shown in Fig. 1. Ethical approval was obtained from the Ethical Review Board of the University Hospitals Leuven (S58253).

\section{Results}

1. Segmentation

The Digital Imaging and Communications in Medicine (DICOM) images of the patient are imported into the Mimics Inprint software (Materialise) for the segmentation step. Image segmentation is the process of assigning voxels with shared characteristics to an object. For VSP, the segmented objects are typically the full skull, the airway and the soft tissue and the segmentation is threshold based. The software tools allow separating the mandible 


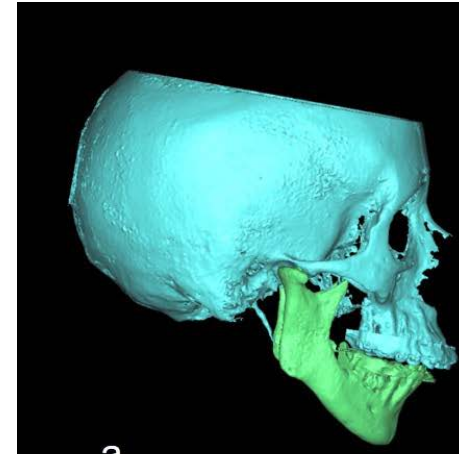

a

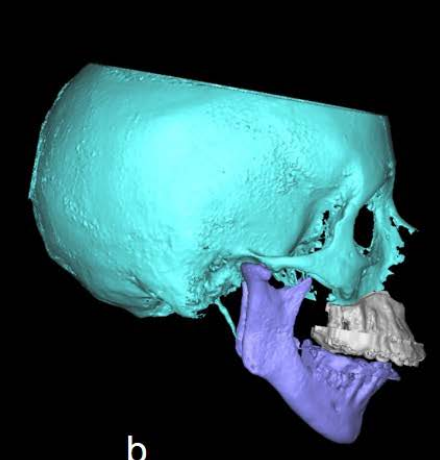

b

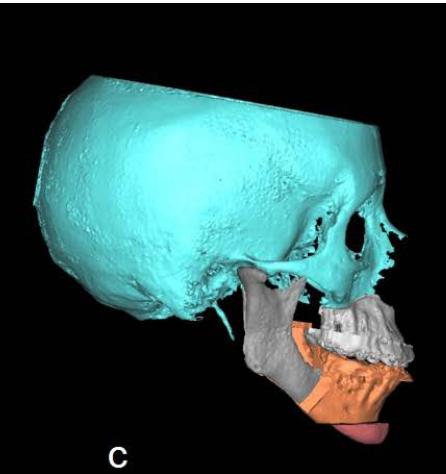

C

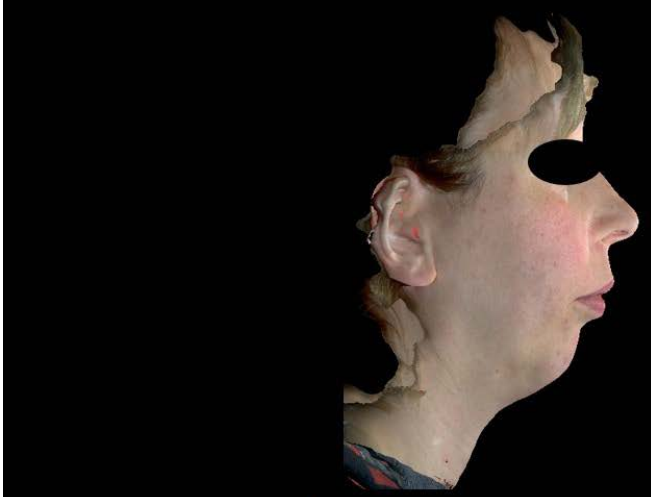

d

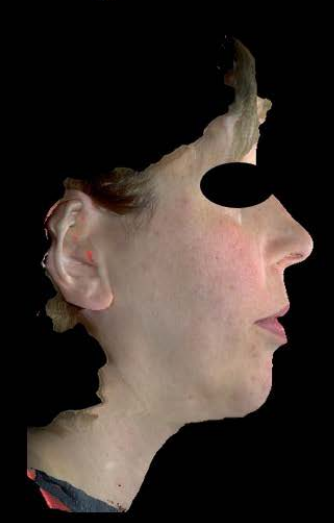

e

Figure 1. An example of a patient undergoing bimaxillary orthognathic surgery:a) the preoperative situation (steps 1 to 3); b) the intermediate situation (clinical plan was: Le Fort ladvancement $1 \mathrm{~mm}$ and translation $2 \mathrm{~mm}$ to the left); c) the final situation with registered $\mathrm{BSSO}$ and genioplasty advancement $6 \mathrm{~mm}$; d) the preoperative 3D photo; e) the soft tissue simulation (step 9).

from the rest of the skull semi-automatically and also refining the segmentation of each object as needed. For example when the condyles are not fully segmented, 3D interpolation tools can be applied to add the missing parts or when some artifacts are presented in the segmented objects, these can be manually removed. 3D models are then reconstructed and exported to the Proplan software. 1. Augmented models

Since the slice thickness of CBCT scans of a full skull is typically within the range of $0.3-1 \mathrm{~mm}$, along with the artefacts introduced into the images due to the orthodontic brackets, the teeth cannot be used later for the fabrication of the splints. Therefore, high resolution scans of the upper and lower teeth are superimposed on the maxilla and the mandible respectively to create augmented models (maxillary and mandibular). The high resolution scans of the teeth can either be the plaster models scanned or direct scan using an intra oral scanner. Either way the output has to be 2 stereolithography (STL) files that are imported into the software and superimposed on the CBCT teeth using surface based registration (Fig. 1a).

\section{Natural Head Position (NHP)}

NHP is allowed in the software using three possibilities: manually, Frankfurt horizontal plane (FHP) or occlusal plane. In our protocol, we start with FHP then manually adjusted according to the clinical images of the patient.

\section{Cephalometric analysis}

The Leuven cephalometric analysis is used which is based on selected measurements of different analyses to analyze the hard tissue, soft tissue, proportions, angular and linear measurements. This analysis was previously developed in $2 \mathrm{D}$ and called Genk Surgical then adopted and extended into 3D. The landmarks, planes and measurements are once created. The software guides the user to place the landmarks then the measurements are automatically calculated and shown on the 3D models.

4. Nerve tracing

For patients undergoing a bilateral sagittal split osteotomy (BSSO), the mandibular inferior alveolar nerves are traced to assist the surgeon with the decision of handling the nerve during the surgery [10].

5. Osteotomy simulation

In the orthognathic wizard, the commonly used osteotomies such as Le Fort 1, BSSO, genioplasty are implemented with a user friendly guide to place specific points then press apply and your objects are cut according to the chosen osteotomy. Furthermore, other osteotomies are allowed using the curve tool in which the user can draw curved planes. This is commonly used for multiple pieces Le Fort 1 or any type of segmental osteotomies.

\section{Occlusion registration}

The STL of the occlusion cast is then imported into the software and registered using surface based registration on the maxilla then the BSSO mandibular 
segment is registered onto the registered cast as explained by Shaheen et al. [11,12]. This order is followed for BSSO and bimaxillary cases. As for a single Le Fort 1 operation, the registration of the cast starts with the mandible then the maxilla is registered to the registered cast. The occlusion cast is the final required occlusion defined and set by the surgeon which can be the plaster cast models in final occlusion scanned by a high resolution $C B C T$ or the printed models from the intra oral scanner scanned once more in final occlusion.

\section{Virtual planning}

For bimaxillary surgery, 3 objects are moved together (Le Fort 1, BSSO and registered occlusion cast). Manual or defined translations and rotations are allowed. Choices of rotations around cephalometric points are also possible. There is freedom of choice while visualizing the amount of movements on the selected cephalometric points (Fig. 1b,c).

\section{Soft tissue simulation}

Once the bony parts are displaced, the soft tissue simulation can be activated and immediately shown. The software allows visualizing the changes occurring on the soft tissue live while changing the bony structures which facilitates updating or improving the final plan. The soft tissue simulation algorithm is based on an improved finite element model [13]. Another advantage is the possibility to visualize the soft tissue simulation on a $3 \mathrm{D}$ photo which is superimposed on the soft tissue segmented object using a combination of landmarks followed by surface based registration (Fig. 1d,e).

3. Splints design

The final step of the orthognathic wizard is to design the splints. For single jaw operation, one final splint is needed. For double jaw surgery, a final and an intermediate splints are needed. The splint is designed by means of placing minimum three points on the upper jaw and three on the lower jaw. Then a first design is presented that can be further refined according to the need of the surgeon. Holes and different inclinations can also be implemented. The splints are then labeled and exported as STL files to be 3D printed.

\section{Discussion}

In this paper, we presented the protocol used in Leuven for VSP of orthognathic surgery which is also in line with the general VSP protocol recently used worldwide. Proplan is our choice for VSP but there are other software available on the market that follow the same protocol. However, in some detailed steps some software could be faster/slower, more or less accurate, etc. Some software tools limit the user to specific scanning protocols to automate the preprocessing steps for faster use, but, with more complex situations it could ask the user to manually interfere to solve some problems. Other software allow exporting the STL files to only the splints which limits the ability of the user to $3 D$ print other objects or to experiment with other technologies such as pre-bended metal plates. Soft tissue simulation is an important tool even though still not completely accurate especially around the nose and lips regions. However, it provides an estimate of what the patient will look like postoperatively, especially patients with obvious facial asymmetry. Some software does not allow superimposing 3D photos which makes it difficult to show the patients the simulation of their postoperative approximate situation due to lack of reality. A software without a clear wizard of the steps is not a strong user friendly tool. A wizard decreases human error as the user follows instructions. The less interference from the user, the more accurate the results will be therefore, more automated tools are needed but refinement tools are strongly recommended to overcome complex situations. Automatic final occlusion should also be implemented in the software but again with the possibility to perform manual corrections. Unfortunately not all software tools allow more than the predefined osteotomies which limits the use of the software for complex cases. Moreover, the lack of segmentation and only allowing visualization threshold restricts the capabilities of the software. After two validation studies $[11,12]$ introducing improvements into the protocol, this specific protocol was implemented on 500 orthognathic patients from 2016. Prior to 2016, another software was used for only simulation purposes without using 3D printed splints and preoperative scans were CT based. An in-house tool was implemented to evaluate the accuracy of the achieved outcome versus the virtually planned maxilla for bimaxillary cases [14] After testing on 55 skeletal class III patients, we concluded that our 3D VSP of maxilla was generally accurate when compared to the outcome achieved [15]. Both advantages and disadvantages need to be recognized. According to our experience in over 500 patients, the following advantages are clinically relevant:

- the 3D-reconstructed virtual head set is a great communication tool to be used when explaining the planned movements to the patient

- the introduction of the virtual planning process has profoundly influenced the life-cycle of the learning process in orthognathic surgery. The ease of superimposition allows the surgeon quickly to understand which distances and which directions of change are difficult to deliver peroperatively.

- postoperative problems with wafers can easily be solved by reprinting the stored STL-file of the wafer - as the original position of the ascendic ramus can be visualized during the entire planning process, lingual bony interferences distal to the tooth bearing area can be readily identified and anticipated, mainly during rotations of the lower jaw, as well as any bone grafting needs in the osteotomy gaps.

- the influence of the surgical plan on the airway can 
- readily be predicted during the planning process

- refinements to the planning concerning pitch, roll and yaw are taken more often into consideration in the digitized planning process.

- it is the only way towards waferless planning with preprinted osteosynthesis plates and it allows the surgeons the freedom to choose between wafer and waferless transfer of the virtual surgical planning to the operating room

- the planning process allows mirroring techniques in hemimandibular or hemifacial hypoplasia to predict the amount of bone/soft tissue needed to reach symmetry.

- severe asymmetry cases are poorly planned in conventional planning tools where the lateral cephalogram remains the main starting file; VSP eliminates this shortcoming as the frontal plane is equally visualized as the sagittal plane

- cutting guides can be designed in delicate surgical osteotomy lines where the inferior alveolar nerve could be compromised.

- during the VSP the position of the condyles is checked and compared, readily identifying incongruences

According to our experience the following disadvantages need to be recognized:

- since the soft tissue changes at the area of the nose are poorly predicted, the amount of advancement of the maxilla remains a decision of mere clinical judgement

- the sagittal position of the maxilla remains as accurate as the initial waxbite. If the waxbite which had been used during planning was erroneous, the sagittal position of the maxilla will be erroneous too if wafers are used

- mild canting of the occlusal plane or upper midline deviations tend to be corrected in respect of the bony 3D reference lines instead of being judged against the soft tissues of the upper lip and nose.

- in multisegmental osteotomies of the upper jaw this digital planning process in no way facilitates designing transpalatal arches in order to stabilize the transverse dimension.

- the cost of software purchase, maintenance and upgrade is considerable and limits its in-house use only to high volume orthognathic practices.

- the workflow does not allow anticipatind nor predicting postoperative condylar resorption, nor temporomandibular joint dysfunction

\section{References}

1. Stokbro $K$, Aagaard $E$, Torkov $P$, et al. Virtual planning in orthognathic surgery. Int J Oral Maxillofac Surg. 2014;43(8):957-965.

[Full text links] [PubMed] Google Scholar Scopus

2. Steinhuber T, Brunold S, Gärtner $C$, et al. Is virtual surgical planning in orthognathic surgery faster than conventional planning? A time and workflow analysis of an office-based workflow for singleand double-jaw surgery. J Oral Maxillofac Surg. 2018;76(2):397-407.

[Full text links] [CrossRef] [PubMed] Google Scholar

3. Schneider D, Kämmerer PW, Hennig $M$, et al. Customized virtual surgical planning in bimaxillary orthognathic surgery: a
- presurgical planning with plaster casts allows the surgeon to easily identify which teeth need to be ground during or before surgery. This is a far more difficult exercise with VSP.

- if scanning of dental casts is used, the errors of plaster dental casts will be transferred into the VSP Virtual surgical planning is also prone to intrinsic weaknesses which urge for validation of methods used:

- 3D-CBCT superimposition algorithms are based on a variety of methods, each with their advantages and disadvantages, but hardly any of them can be considered accurate in growing individuals; different segmentation techniques can result in significant variances [16].

- the resulting differences in volume usually are depicted in color-maps, representing colorcoded surface distances, allowing a qualitative interpretation of the changes but hardly any robust volumetric changes.

- many methods of superimposition using other anatomical structures than the mandible to study condylar resorption after orthognathic surgery fail to be accurate due to possible changes of condylar position between the two measurements.

A number of advantages of the availability of CBCT-data should not be attributed to VSP, such as the traceability of the inferior alveolar nerve, the positioning of the condyle in the fossa, the follow-up of qualitative changes of the condyle.

\section{Conclusion}

Virtual Surgical Planning can safely replace Custom Manual Surgical Planning but does not constitute a major advantage if its use is limited to digital splint design and production. It is a necessary step towards a renewed orthognathic planning process where the bony changes are reliably deducted from the desired functional and esthetic orthognathic outcome using validated processes and methods.

\section{Author contributions}

All authors contributed to reporting the case, performing literature review and drafting the manuscript.

\section{Conflict of interest}

No conflict of interest to declare. prospective randomized trial. Clin Oral Investig. 2019;23(7):31153122.

[Full text links] [CrossRef] [PubMed] Google Scholar Scopus

4. Gateno J, Xia JJ, Teichgraeber JF, et al. Clinical feasibility of computer-aided surgical simulation (CASS) in the treatment of complex cranio-maxillofacial deformities. J Oral Maxillofac Surg. 2007;65(4):728-734.

[Full text links] [CrossRef] [PubMed] Google Scholar

5. Xia JJ, Gateno J, Teichgraeber JF, et al. Algorithm for planning a double-jaw orthognathic surgery using a computer-aided 
cephalometry. Int J Oral Maxillofac Surg. 2015;44(12):1441-1450.

[Full text links] [CrossRef] [PubMed] Google Scholar

6. Farrell BB, Franco PB, Tucker MR. Virtual surgical planning in orthognathic surgery. Oral Maxillofac Surg Clin North Am. 2014;26(4):459-473.

[Full text links] [PubMed] Google Scholar Scopus

7. Swennen GR, Mollemans W, Schutyser F. Three-dimensional treatment planning of orthognathic surgery in the era of virtual imaging. J Oral Maxillofac Surg. 2009;67(10):2080-2092.

[Full text links] [CrossRef] [PubMed] Google Scholar Scopus

8. Centenero SA, Hernández-Alfaro F. 3D planning in orthognathic surgery: CAD/CAM surgical splints and prediction of the soft and hard tissues results-our experience in 16 cases. J Craniomaxillofac Surg. 2012;40(2):162-168.

[Full text links] [CrossRef] [PubMed] Google Scholar Scopus

9. Swennen GR, Mollemans W, De Clercq C, et al. A cone-beam computed tomography triple scan procedure to obtain a three-dimensional augmented virtual skull model appropriate for orthognathic surgery planning. J Craniofac Surg. 2009;20(2):297-307. [Full text links] [CrossRef] [PubMed] Google Scholar Scopus 10. Agbaje JO, Sun Y, De Munter S, et al. CBCT-based predictability of attachment of the neurovascular bundle to the proximal segment of the mandible during sagittal split osteotomy. Int J Oral Maxillofac Surg. 2013;42(3):308-315.

[Full text links] [CrossRef] [PubMed] Google Scholar Scopus
11. Shaheen $E$, Sun $Y$, Jacobs $R$, Politis C. Three-dimensional printed final occlusal splint for orthognathic surgery: design and validation. Int J Oral Maxillofac Surg. 2017;46(1):67-71.

[Full text links] [CrossRef] [PubMed] Google Scholar Scopus

12. Shaheen E, Coopman R, Jacobs R, Politis C. Optimized $3 \mathrm{D}$ virtually planned intermediate splints for bimaxillary orthognathic surgery: A clinical validation study in 20 patients. $J$ Craniomaxillofac Surg. 2018;46(9):1441-1447.

[Full text links][CrossRef][PubMed] Google Scholar Scopus

13. Marchetti C, Bianchi A, Muyldermans L, et al. Validation of new soft tissue software in orthognathic surgery planning. Int J Oral Maxillofac Surg. 2011;40(1):26-32.

[Full text links] [CrossRef] [PubMed] Google Scholar Scopus

14. Shaheen E, Shujaat S, Saeed T, et al. Three-dimensional planning accuracy and follow-up protocol in orthognathic surgery: a validation study. Int J Oral Maxillofac Surg. 2019;48(1):71-76.

[Full text links] [CrossRef] [PubMed] Google Scholar Scopus

15. Mulier D, Shaheen E, Shujaat S, et al. How accurate is digitalassisted Le Fort I maxillary osteotomy? A three-dimensional perspective. Int J Oral Maxillofac Surg. 2019. pii: S09015027(19)31186-5.

[Full text links] [CrossRef] [PubMed] Google Scholar

16. Park JH, Tai K, Owtad P. 3-Dimensional cone-beam computed tomography superimposition: A review. Semin Orthod. 2015;21(4):263-273.

[CrossRef] Google Scholar Scopus

\section{Eman SHAHEEN \\ BSc, MSc, PhD, Clinical Engineer \\ Responsible 3D Surgical Orthognathic Lab Department of Oral and Maxillofacial Surgery University Hospitals Leuven BE-3000 Leuven, Belgium}

Eman Shaheen graduated with honor from the faculty of Computer Sciences (2003), Cairo University, Egypt where she worked as a teaching assistant till 2007 when she obtained her Master's Degree in Video Processing. In 2008, she joined the team of Medical Physics where she completed with distinction her pre-doctoral studies (2009) followed by her doctoral degree (2014) after developing a simulation framework to optimize the performance of breast tomosynthesis, KU Leuven, Belgium. In the same year, she started working for the CMF department (University hospitals Leuven, Belgium) as a clinical engineer focusing on 3D planning of orthognathic surgery. She is also the lead engineer of the research group "OMFSIMPATH" (KU Leuven, Belgium) where she supervises masters and PhD students to support different research projects related to 3D printing and simulations.

\section{0restions}

\section{The software used by UZ Leuven for VSP is:}

a. Proplan;

口b. Simplant O\&O;

口c. IPS;

$\square d$. Mimics.

\section{The UZ Leuven protocol was implemented on over:}

口a. 50 patients;

b. 150 patients;

口. 300 patients;

dd. 500 patients.

\section{One of the following is considered an advantage of VSP:}

$\square$ a. Dependency on initial waxbite;

b. Registering occlusion casts;

ac. Reprint of STL of wafers;

$\square d$. Nerve tracing.

\section{One of the following is considered a disadvantage of VSP:}

$\square$ a. Dependency on initial waxbite;

b. Registering occlusion casts;

ac. Reprint of STL of wafers;

$\square$ d. Nerve tracing. 